\title{
Vibration of Visco-Elastic Parallelogram Plate with Parabolic Thickness Variation
}

\author{
Arun Kumar Gupta ${ }^{*}$, Anuj Kumar, Yogesh Kumar Gupta \\ Maharaj Singh College, Saharanpur, India \\ E-mail:gupta_arunnitin@yahoo.co.in \\ Received April 7, 2010; revised May 10, 2010; accepted May 17, 2010
}

\begin{abstract}
The main objective of the present investigation is to study the vibration of visco-elastic parallelogram plate whose thickness varies parabolically. It is assumed that the plate is clamped on all the four edges and that the thickness varies parabolically in one direction i.e. along length of the plate. Rayleigh-Ritz technique has been used to determine the frequency equation. A two terms deflection function has been used as a solution. For visco-elastic, the basic elastic and viscous elements are combined. We have taken Kelvin model for visco-elasticity that is the combination of the elastic and viscous elements in parallel. Here the elastic element means the spring and the viscous element means the dashpot. The assumption of small deflection and linear visco-elastic properties of "Kelvin" type are taken. We have calculated time period and deflection at various points for different values of skew angles, aspect ratio and taper constant, for the first two modes of vibration. Results are supported by tables. Alloy "Duralumin" is considered for all the material constants used in numerical calculations.
\end{abstract}

Keywords: Vibration, Parallelogram Plate, Visco-Elastic Mechanics, Parabolic Thickness, Aspect Ratio

\section{Introduction}

The materials are being developed, depending upon the requirement and durability, so that these can be used to give better strength, flexibility, weight effectiveness and efficiency. So some new materials and alloys are utilized in making structural parts of equipment used in modern technological industries like space craft, jet engine, earth quake resistance structures, telephone industry etc. Applications of such materials are due to reduction of weight and size, low expenses and enhancement in effectiveness and strength. It is well known that first few frequencies of structure should be known before finalizing the design of a structure. The study of vibration of skew plate structures is important in a wide variety of applications in engineering design. Parallelogram elastic plates are widely employed nowadays in civil, aeronautical and marine structures designs. Complex shapes with variety of thickness variation are sometimes incorporated to reduce costly material, lighten the loads, and provide ventilation and to alter the resonant frequencies of the structures. Dynamic behavior of these structures is strongly dependent on boundary conditions, geometric shapes, material properties etc.
Dhotarad and Ganesan [1] have considered vibration analysis of a rectangular plate subjected to a thermal gradient. Amabili and Garziera [2] have studied transverse vibrations of circular, annular plates with several combinations of boundary conditions. Ceribasi and Altay [3] introduced the free vibration analysis of super elliptical plates with constant and variable thickness by Ritz method. Gupta, Ansari and Sharma [4] have analyzed vibration analysis of non-homogenous circular plate of non linear thickness variation by differential quadrature method. Jain and Soni [5] discussed free vibrations of rectangular plates of parabolically varying thickness. Singh and Saxena [6] discussed the transverse vibration of rectangular plate with bi-directional thickness. Free vibrations of non-homogeneous circular plate of variable thickness resting on elastic foundation are discussed by Tomar, Gupta and Kumar [7]. Yang [8] has considered the vibration of a circular plate with varying thickness. Gupta, Ansari and Sharma [9] discussed the vibration of non-homogeneous circular Mindlin plates with variable thickness. Bambill, Rossit, Laura and Rossi [10] have analyzed transverse vibration of an orthotropic rectangular plate of linearly varying thickness with a free edge.

Sufficient work $[11,12]$ is available on the vibration of a rectangular plate of variable thickness in one direction, 
but none of them done on parallelogram plate. Recently Gupta, Kumar and Gupta [13] studied the vibration of visco-elastic parallelogram plate of linearly varying thickness. A simple model presented here is to study the effect of parabolic thickness variation on vibration of viscoelastic parallelogram plate having clamped boundary conditions on all the four edges. The hypothesis of small deflection and linear visco-elastic properties are made. Using the separation of variables method, the governing differential equation has been solved for vibration of visco-elastic parallelogram plate. An approximate but quite convenient frequency equation is derived by using Rayleigh-Ritz technique with a two-term deflection function. It is assumed that the visco-elastic properties of the plate are of the "Kelvin Type". Time period and deflection function at different point for the first two modes of vibration are calculated for various values of taper constant, aspect ratio and skew angle and results are presented in tabular form.

\section{Equation of Transverse Motion}

The parallelogram (skew) plate is assumed to be nonuniform, thin and isotropic and the plate $R$ be defined by the three number $a, b$ and $\theta$ as shown in Figure 1.

The skew coordinates are related to rectangular coordinates are

$$
\xi=x-y \tan \theta, \eta=y \sec \theta
$$

The boundaries of the plate in skew coordinates are

$$
\xi=0, \xi=a, \eta=0, \eta=b
$$

The governing differential equation of transverse motion of visco-elastic parallelogram plate of variable thickness, $\xi$ - and $\eta$-coordinates is given by [13]:

$$
T_{\max }=\frac{1}{2} \rho p^{2} \cos \theta \iint h W^{2} d \xi d \eta
$$

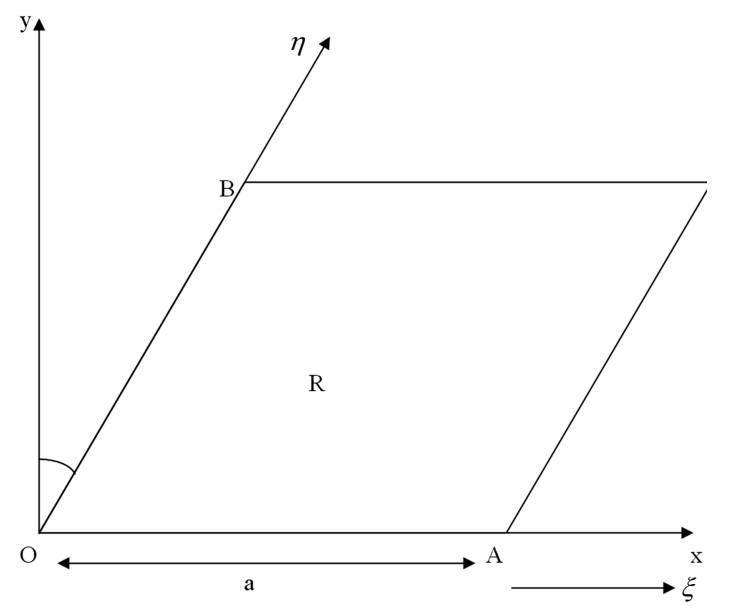

Figure 1. Geometry of parallelogram plate. and

$$
\begin{aligned}
V= & \frac{1}{2} \cos \theta \iint D\left[\left(\nabla^{2} W\right)^{2}-2(1-v) \cdot\right. \\
& \left.\sec ^{2} \theta\left(W,{ }_{\xi \xi} W,{ }_{\eta \eta}-W,{ }_{\xi \eta}^{2}\right)\right] d \xi d \eta
\end{aligned}
$$

or

$$
\begin{aligned}
& V=\frac{1}{2 \cos ^{3} \theta} \iint D\left[W_{, \xi \xi}{ }^{2}-4 \sin \theta W_{, \xi \xi} W_{, \xi \eta}+2\left(\sin ^{2} \theta\right.\right. \\
& \left.+v \cos ^{2} \theta\right) W_{, \xi \xi} W_{, \eta \eta}+2\left(1+\sin ^{2} \theta-v \cos ^{2} \theta\right) \text {. } \\
& \left.W_{, \xi \eta}{ }^{2}-4 \sin \theta W_{, \xi \eta} W_{, \eta \eta}+W_{, \eta \eta}{ }^{2}\right] d \xi d \eta
\end{aligned}
$$

and

$$
T_{, t t}+p^{2} \check{\mathrm{D}} T=0
$$

A comma followed by a suffix denotes partial differential with respect to that variable. Here $p^{2}$ is a constant.

Here solution $w(\xi, \eta, t)$ can be taken in the form of products of two functions as for free transverse vibration of the parallelogram plate so that

$$
w(\xi, \eta, t)=W(\xi, \eta) T(t)
$$

where $T(t)$ is the time function and $W$ is the maximum displacement with respect to time $t$.

Assuming thickness variation of parallelogram plate parabolically in $\xi$-direction as

$$
h=h_{0}\left\{1+\beta(\xi / a)^{2}\right\}
$$

where $\beta$ is the taper constant in $\xi$-direction and $h_{0}=$ $\left.h\right|_{\xi=0}$.

The flexural rigidity $D$ of the plate can now be written as

$$
D=E h_{0}^{3}\left(1+\beta(\xi / a)^{2}\right)^{3} / 12\left(1-v^{2}\right)
$$

\section{Solution and Frequency Equation}

In using the Rayleigh-Ritz technique, one requires maximum strain energy be equal to the maximum kinetic energy. So it is necessary for the problem consider here that

$$
\delta\left(V-T_{\max }\right)=0
$$

for arbitrary variations of $W$ satisfying relevant geometrical boundary conditions.

For a parallelogram plate, clamped $(c)$ along all the four edges, the boundary conditions are

$$
\begin{aligned}
& W=W_{, \xi}=0 \text { at } \xi=0, a \text { and } \\
& W=W_{, \eta}=0 \text { at } \eta=0, b
\end{aligned}
$$

and the corresponding two-term deflection function is taken as 


$$
\begin{aligned}
W= & {[(\xi / a)(\eta / b)(1-\xi / a)(1-\eta / b)]^{2} . } \\
& {\left[A_{1}+A_{2}(\xi / a)(\eta / b)(1-\xi / a)(1-\eta / b)\right] }
\end{aligned}
$$

Using Equations (5) and (6) in Equations (1) and (2), one obtains

$$
T_{\max }=\frac{1}{2} h_{0} \rho p^{2} \cos \theta \int_{\eta=0}^{b} \int_{\xi=0}^{a}\left(1+\beta\left(\frac{\xi}{a}\right)^{2}\right) W^{2} d \xi d \eta
$$

and

$$
\begin{aligned}
V= & \frac{E h_{0}^{3}}{24\left(1-v^{2}\right) \cos ^{3} \theta} \int_{\eta=0}^{b} \int_{\xi=0}^{a}\left(1+\beta\left(\frac{\xi}{a}\right)^{2}\right)^{3}\left[W_{, \xi \xi}^{2}-4\left(\frac{a}{b}\right)\right. \\
& \sin \theta \mathrm{W}_{, \xi \xi} \mathrm{W}_{, \xi \eta}+2\left(\frac{a}{b}\right)^{2}\left(\sin ^{2} \theta+v \cos ^{2} \theta\right) \mathrm{W}_{, \xi \xi} \mathrm{W}_{, \eta \eta} \\
& +2\left(\frac{a}{b}\right)^{2}\left(1+\sin ^{2} \theta-v \cos ^{2} \theta\right) \mathrm{W}_{, \xi \eta}{ }^{2}-4\left(\frac{a}{b}\right)^{3} \\
& \left.\sin \theta \mathrm{W}_{, \xi \eta} \mathrm{W}_{, \eta \eta}+\left(\frac{a}{b}\right)^{4} \mathrm{~W}_{, \eta \eta}{ }^{2}\right] d \xi d \eta
\end{aligned}
$$

Using Equations (10) and (11) in Equation (7), one obtains

$$
\left(V_{1}-\lambda^{2} p^{2} T_{1}\right)=0
$$

where

$$
\begin{aligned}
V_{1}= & \int_{\eta=0}^{b} \int_{\xi=0}^{a}\left(1+\beta\left(\frac{\xi}{a}\right)^{2}\right)^{3}\left[W_{, \xi \xi}^{2}-4\left(\frac{a}{b}\right) \cdot\right. \\
& \sin \theta \mathrm{W}_{, \xi \xi} \mathrm{W}_{, \xi \eta}+2\left(\frac{a}{b}\right)^{2}\left(\sin ^{2} \theta+v \cos ^{2} \theta\right) \mathrm{W}_{, \xi \xi} \mathrm{W}_{, \eta \eta} \\
& +2\left(\frac{a}{b}\right)^{2}\left(1+\sin ^{2} \theta-v \cos ^{2} \theta\right) \mathrm{W}_{, \xi \eta}^{2}-4\left(\frac{a}{b}\right)^{3} \\
& \left.\sin \theta \mathrm{W}_{, \xi \eta} \mathrm{W}_{, \eta \eta}+\left(\frac{a}{b}\right)^{4} \mathrm{~W}_{, \eta \eta}^{2}\right] d \xi d \eta
\end{aligned}
$$

and

$$
T_{1}=\cos ^{4} \theta \int_{0}^{b} \int_{0}^{a}\left(1+\beta\left(\frac{\xi}{a}\right)^{2}\right) W^{2} d \xi d \eta
$$

Here,

$$
\lambda^{2}=\frac{12 a^{4} \rho\left(1-v^{2}\right)}{E h_{0}^{2}}
$$

is a constant.

But Equation (12) involves the unknown $A_{1}$ and $A_{2}$ arising due to the substitution of $W(\xi, \eta)$ from Equation (9). These two constants are to be determined from Equation (12), as follows:

$$
\partial\left(V_{1}-\lambda^{2} p^{2} T_{1}\right) / \partial A_{n}=0, \quad n=1,2
$$

Equation (16) simplifies to the form

$$
b_{n 1} A_{1}+b_{n 2} A_{1}=0, \quad n=1,2
$$

where $b_{n 1}, b_{n 2}(n=1,2)$ involve parametric constants and the frequency parameter.

For a non-trivial solution, the determinant of the coefficient of Equation (17) must be zero. So one gets the frequency equation as

$$
\left|\begin{array}{ll}
b_{11} & b_{12} \\
b_{21} & b_{22}
\end{array}\right|=0
$$

Here,

$$
\begin{aligned}
& b_{11}=\left(F_{1}-\lambda^{2} p^{2} B_{1}\right), \\
& b_{12}=b_{21}=\left(F_{2}-\lambda^{2} p^{2} B_{2}\right), \\
& b_{22}=\left(F_{3}-\lambda^{2} p^{2} B_{3}\right)
\end{aligned}
$$

and

where $F_{1}, F_{2}, F_{3}, B_{1}, B_{2}, B_{3}$ involves parametric constants, skew angle and aspect ratio and given as

$$
\begin{aligned}
F_{1}= & I_{1}+2\left(\frac{a}{b}\right)^{2}\left(\sin ^{2} \theta+v \cos ^{2} \theta\right) I_{2}+2\left(\frac{a}{b}\right)^{2}\left(1+\sin ^{2} \theta\right. \\
& \left.-v \cos ^{2} \theta\right) I_{3}+\left(\frac{a}{b}\right)^{4} I_{4}, \\
B_{1}= & \cos ^{4} \theta I_{5}, \\
F_{2}= & I_{6}+2\left(\frac{a}{b}\right)^{2}\left(\sin ^{2} \theta+v \cos ^{2} \theta\right) I_{7}+2\left(\frac{a}{b}\right)^{2}\left(1+\sin ^{2} \theta\right. \\
& \left.-v \cos ^{2} \theta\right) I_{8}+\left(\frac{a}{b}\right)^{4} I_{9}, \\
B_{2}= & \cos ^{4} \theta I_{10}, \\
F_{3}= & I_{11}+2\left(\frac{a}{b}\right)^{2}\left(\sin ^{2} \theta+v \cos ^{2} \theta\right) I_{12}+2\left(\frac{a}{b}\right)^{2}\left(1+\sin ^{2} \theta\right. \\
& \left.-v \cos ^{2} \theta\right) I_{13}+\left(\frac{a}{b}\right)^{4} I_{14}, \\
B_{3}= & \cos ^{4} \theta I_{15},
\end{aligned}
$$

Here,

$$
\begin{aligned}
& I_{1}=\frac{2}{1575}+\frac{16}{11025} \beta+\frac{4}{3675} \beta^{2}+\frac{113}{363825} \beta^{3}, \\
& I_{2}=\frac{4}{11025}+\frac{1}{3675} \beta+\frac{8}{121275} \beta^{2}+\frac{2}{675675} \beta^{3}, \\
& I_{3}=\frac{4}{11025}+\frac{1}{3675} \beta+\frac{8}{121275} \beta^{2}+\frac{2}{675675} \beta^{3}, \\
& I_{4}=\frac{2}{1575}+\frac{2}{1925} \beta+\frac{4}{10725} \beta^{2}+\frac{4}{75075} \beta^{3}, \\
& I_{5}=\frac{1}{396900}+\frac{1}{1455300} \beta, \\
& I_{6}=\frac{1}{8085}+\frac{1}{5390} \beta+\frac{4}{53361} \beta^{2}+\frac{37}{1981980} \beta^{3}, \\
& I_{7}=\frac{1}{22050}+\frac{4}{121275} \beta+\frac{1}{150150} \beta^{2}+\frac{1}{194040} \beta^{3},
\end{aligned}
$$




$$
\begin{aligned}
I_{8}= & \frac{1}{22050}+\frac{1}{23100} \beta+\frac{1}{42900} \beta^{2}+\frac{1}{210210} \beta^{3}, \\
I_{9}= & \frac{1}{8085}+\frac{1}{10010} \beta+\frac{6}{175175} \beta^{2}+\frac{1}{216580} \beta^{3}, \\
I_{10}= & \frac{1}{3841992}+\frac{1}{14270256} \beta, \\
I_{11}= & \frac{1}{210210}+\frac{1}{220220} \beta+\frac{5}{2004002} \beta^{2} \\
& +\frac{17}{30060030} \beta^{3}, \\
I_{12}= & \frac{1}{592900}+\frac{19}{15415400} \beta+\frac{1}{3853850} \beta^{2} \\
& +\frac{3}{524123600} \beta^{3}, \\
I_{13}= & \frac{1}{592900}+\frac{3}{1926925} \beta+\frac{3}{3853850} \beta^{2} \\
& +\frac{3}{20158600} \beta^{3}, \\
I_{14}= & \frac{1}{210210}+\frac{2}{525525} \beta+\frac{3}{2382380} \beta^{2} \\
& +\frac{1}{6172530} \beta^{3}, \\
I_{15}= & \frac{1}{144288144}+\frac{1}{541080540} \beta,
\end{aligned}
$$

From Equation (18), one can obtains a quadratic equation in $p^{2}$ from which the two values of $p^{2}$ can found. After determining $A_{1} \& A_{2}$ from Equation (17), one can obtain deflection function $W$. Choosing $A_{1}=1$, one obtains $A_{2}=\left(-b_{11} / b_{12}\right)$ and then $W$ comes out as

$$
\begin{aligned}
W= & {[X Y(a / b)(1-X)(1-Y a / b)]^{2} } \\
& {\left[1+\left(-b_{11} / b_{12}\right) X Y(a / b)(1-X)(1-Y a / b)\right] . }
\end{aligned}
$$

Here

$$
X=\xi / a \text { and } Y=\eta / a
$$

\section{Differential Equation of Time Function and its Solution}

Time functions of free vibrations of viscoelastic plates are defined by the general ordinary differential Equation (3). Their form depends on the viscoelastic operator $\check{D}$. For Kelvin's model, one has

$$
\check{D}=\{1+(\check{n} / G)(d / d t)\}
$$

where, $\check{n}$ is viscoelastic constant and $G$ is shear modulus.

The governing differential equation of time function of a parallelogram plate of variable thickness, by using Equation (20) in Equation (3), one obtains as

$$
T_{, t t}+p^{2}(\check{n} / G) T_{, t}+p^{2} T=0
$$

Equation (21) is a differential equation of order two for time function $T$. Solution of Equation (21) comes out as

$$
T(t)=e^{k t}\left(C_{1} \cos k_{1} t+C_{1} \sin k_{1} t\right)
$$

where,

$$
k=-p^{2} \check{n} / 2 G
$$

and

$$
k_{1}=p\left\{1-(p \check{n} / 2 G)^{2}\right\}^{1 / 2}
$$

Let us take initial conditions as

$$
T=1 \text { and } d T / d t=0 \text { at } t=0
$$

Using initial conditions from Equation (25) in Equation (22), one obtains

$$
T(t)=e^{k t}\left[\cos \left(k_{1} t\right)+\left(-k / k_{1}\right) \sin \left(k_{1} t\right)\right]
$$

Thus, deflection w may be expressed, by using Equations (26) and (19) in Equation (4), to give

$$
\begin{aligned}
& W=[X Y(a / b)(1-X)(1-Y a / b)]^{2} . \\
& {\left[1+\left(-b_{11} / b_{12}\right) X Y(a / b)(1-X)(1-Y a / b)\right] .} \\
& {\left[e^{k t}\left\{\cos \left(k_{1} t\right)\right\}+\left(-k / k_{1}\right) \sin \left(k_{1} t\right)\right]}
\end{aligned}
$$

Time period of vibration of the plate is given by

$$
K=2 \pi / p,
$$

where $p$ is frequency given by Equation (18).

\section{Results and Discussion}

Time period and deflection are computed for visco-elastic parallelogram plate, whose thickness varies parabolically, for different value of skew angle $(\theta)$, taper constant $(\beta)$, and aspect ratio $(a / b)$ at different points for first two mode of vibration. The material parameters have been taken as [14]: $E=7.08 \times 10^{10} \mathrm{n} / \mathrm{m}^{2}, G=2.682 \times 10^{10} \mathrm{n} / \mathrm{m}^{2}, \check{n}=$ $1.4612 \times 10^{6} \mathrm{n} . \mathrm{s} / \mathrm{m}^{2}, \rho=2.80 \times 10^{3} \mathrm{~kg} / \mathrm{m}^{3}, v=0.345$ and $h_{0}=0.01$ meter.

All the results are presented in the Tables 1-11.

The value of time period $(K)$ for $\beta=0.6, \theta=45^{\circ}$ have been found to decrease $35.89 \%$ for first mode and $34.74 \%$ for second mode in comparison to rectangular plate at fixed aspect ratio $(a / b=1.5)$.

The value of time period $(K)$ for $\beta=0.6, \theta=45^{\circ}$ have been found to decrease $20.54 \%$ for first mode and $21.23 \%$ for second mode in comparison to parallelogram plate of uniform thickness at fixed aspect ratio $(a / b=1.5)$.

Table 1 shows the results of time period $(K)$ for different values of taper constant $(\beta)$ and fixed aspect ratio $(a / b=1.5)$ for two values of skew angle $(\theta)$ i.e. $\theta=0^{\circ}$ and $\theta=45^{\circ}$ for first two mode of vibration. It can be seen that the time period $(K)$ decrease when taper constant $(\beta)$ increase for first two mode of vibration.

Table 2 shows the results of time period $(K)$ for dif- 
ferent values of skew angle $(\theta)$ and fixed aspect ratio $(a / b=1.5)$ for two values of taper constant $(\beta)$ i.e. $\beta=$ $0.0, \beta=0.2$ for first two mode of vibration. It can be seen that the time period $(K)$ decrease when skew angle $(\theta)$ increase for first two mode of vibration. .

Table 3 shows the results of time period $(K)$ for different values of aspect ratio $(a / b)$ and fixed taper constant $(\beta=0.0$ and $\beta=0.6)$ for two values of skew angle $(\theta)$ i.e. $\theta=0^{\circ}$ and $\theta=45^{\circ}$ for first two mode of vibration. It can be seen that the time period $(K)$ decrease when aspect ratio $(a / b)$ increase for first two mode of vibration.

The value of deflection $(w)$ for $\beta=0.6$ and $\theta=45^{\circ}$ have been found to increase $14.19 \%$ for first mode and $1.07 \%$ for second mode in comparison to parallelogram plate of uniform thickness for initial time $0 . \mathrm{K}$ at $X=0.2$, $Y=0.4$ and $a / b=1.5$.

The value of deflection $(w)$ for $\beta=0.6$ and $\theta=45^{\circ}$ have been found to decrease $4.76 \%$ for first mode and $0.53 \%$ for second mode in comparison to rectangular plate for initial time $0 . \mathrm{K}$ at $X=0.2, Y=0.4$ and $a / b=$ 1.5 .

The value of deflection $(w)$ for $\beta=0.6$ and $\theta=45^{\circ}$ have been found to increase $11.91 \%$ for first mode and decrease $6.03 \%$ for second mode in comparison to parallelogram plate of uniform thickness for time $5 . \mathrm{K}$ at $X=$

Table 1. Time period $K$ (in second) for different taper constant $(\beta)$ and a constant aspect ratio $(a / b=1.5)$.

\begin{tabular}{ccccc}
\hline & \multicolumn{2}{c}{$\theta=0^{\circ}$} & \multicolumn{2}{c}{$\theta=45^{\circ}$} \\
\cline { 2 - 5 }$\beta$ & $\begin{array}{c}\text { First } \\
\text { Mode }\end{array}$ & $\begin{array}{c}\text { Second } \\
\text { Mode }\end{array}$ & $\begin{array}{c}\text { First } \\
\text { Mode }\end{array}$ & $\begin{array}{c}\text { Second } \\
\text { Mode }\end{array}$ \\
\hline 0.0 & 0.142648 & 0.036357 & 0.090306 & 0.023976 \\
0.2 & 0.131780 & 0.033664 & 0.084639 & 0.022199 \\
0.4 & 0.121820 & 0.030664 & 0.078029 & 0.020376 \\
0.6 & 0.112958 & 0.028060 & 0.072148 & 0.018465 \\
0.8 & 0.104063 & 0.025895 & 0.067140 & 0.017093 \\
\hline
\end{tabular}

$0.2, Y=0.4$ and $a / b=1.5$.

The value of deflection $(w)$ for $\beta=0.6$ and $\theta=45^{\circ}$ have been found to decrease $7.91 \%$ for first mode and $11.96 \%$ for second mode in comparison to rectangular plate for time $5 . \mathrm{K}$ at $X=0.2, Y=0.4$ and $a / b=1.5$.

Tables 4-11 show the results of deflection $(w)$ for different values of $X, Y$ and fixed taper constant $(\beta=0.0$ and $\beta=0.6)$, and aspect ratio $(a / b=1.5)$ for two values of skew angle $(\theta)$ i.e. $\theta=0^{\circ}$ and $\theta=45^{\circ}$ for first two mode of vibration with time $0 . \mathrm{K}$ and 5 .K. It can be seen that deflection $(w)$ start from zero to increase then decrease to zero for first two mode of vibration (except second mode at $Y=0.2$ and 0.4 ) and second mode of vibration deflection $(w)$ at $(Y=0.2$ and $Y=0.4)$ start zero to increase, decrease, increase, decrease and finally become to zero for different value of $X$.

\section{Conclusions}

The Rayleigh-Ritz technique has been applied to study the effect of the taper constants on the vibration of clamped visco-elastic isotropic parallelogram plate with parabolically varying thickness on the basis of classical plate theory.

Table 2. Time period $K$ (in second) for different skew angle $(\theta)$ and a constant aspect ratio $(a / b=1.5)$.

\begin{tabular}{ccccc}
\hline & \multicolumn{2}{c}{$\beta=0.0$} & \multicolumn{2}{c}{$\beta=0.2$} \\
\cline { 2 - 5 }$\theta$ & $\begin{array}{c}\text { First } \\
\text { Mode }\end{array}$ & $\begin{array}{c}\text { Second } \\
\text { Mode }\end{array}$ & $\begin{array}{c}\text { First } \\
\text { Mode }\end{array}$ & $\begin{array}{c}\text { Second } \\
\text { Mode }\end{array}$ \\
\hline $0^{\circ}$ & 0.142648 & 0.036357 & 0.131780 & 0.033664 \\
$15^{\circ}$ & 0.137773 & 0.035762 & 0.127248 & 0.032985 \\
$30^{\circ}$ & 0.121041 & 0.031427 & 0.112004 & 0.029016 \\
$45^{\circ}$ & 0.090306 & 0.023976 & 0.084639 & 0.022199 \\
$60^{\circ}$ & 0.051089 & 0.013211 & 0.047425 & 0.012143 \\
$75^{\circ}$ & 0.014111 & 0.003431 & 0.013235 & 0.003491 \\
\hline
\end{tabular}

Table 3. Time period $K$ (in second) for different aspect ratio (a/b).

\begin{tabular}{|c|c|c|c|c|c|c|c|c|}
\hline \multirow{2}{*}{$a / b$} & \multicolumn{2}{|c|}{$\beta=0.0, \theta=0^{\circ}$} & \multicolumn{2}{|c|}{$\beta=0.0, \theta=45^{\circ}$} & \multicolumn{2}{|c|}{$\beta=0.6, \theta=0^{\circ}$} & \multicolumn{2}{|c|}{$\beta=0.6, \theta=45^{\circ}$} \\
\hline & First Mode & Second Mode & First Mode & Second Mode & First Mode & Second Mode & First Mode & Second Mode \\
\hline 0.5 & 0.172515 & 0.042510 & 0.1189167 & 0.029103 & 0.133024 & 0.032025 & 0.091918 & 0.022024 \\
\hline 1.0 & 0.159377 & 0.040155 & 0.106213 & 0.027055 & 0.124312 & 0.030879 & 0.083221 & 0.020997 \\
\hline 1.5 & 0.142648 & 0.036357 & 0.090306 & 0.023976 & 0.112958 & 0.028060 & 0.072148 & 0.018465 \\
\hline 2.0 & 0.125147 & 0.033092 & 0.075399 & 0.020193 & 0.100177 & 0.026135 & 0.061061 & 0.016145 \\
\hline 2.5 & 0.108096 & 0.028951 & 0.061982 & 0.016358 & 0.087268 & 0.023080 & 0.050393 & 0.013274 \\
\hline
\end{tabular}


Table 4. Deflection $w$ for different $X, Y$ and $\beta=0.0, \theta=0^{\circ}$ and $a / b=1.5$ at initial time $0 . K$.

\begin{tabular}{ccccccccc}
\hline \multirow{2}{*}{$X$} & \multicolumn{2}{c}{$Y=0.2$} & \multicolumn{2}{c}{$Y=0.4$} & \multicolumn{2}{c}{$Y=0.6$} & \multicolumn{2}{c}{$Y=0.8$} \\
\cline { 2 - 9 } & First Mode & Second Mode & First Mode & Second Mode & First Mode & Second Mode & First Mode & Second Mode \\
\hline 0.0 & 0.000000 & 0.000000 & 0.000000 & 0.000000 & 0.000000 & 0.000000 & 0.000000 & 0.000000 \\
0.2 & 0.001085 & 0.000390 & 0.001411 & 0.000371 & 0.000201 & 0.000145 & 0.001533 & 0.002571 \\
0.4 & 0.002398 & 0.000055 & 0.003102 & -0.000385 & 0.000452 & 0.000268 & 0.003522 & 0.007022 \\
0.6 & 0.002398 & 0.000055 & 0.003102 & -0.000385 & 0.000452 & 0.000268 & 0.003522 & 0.007022 \\
0.8 & 0.001085 & 0.000390 & 0.001411 & 0.000371 & 0.000201 & 0.000145 & 0.001533 & 0.002571 \\
1.0 & 0.000000 & 0.000000 & 0.000000 & 0.000000 & 0.000000 & 0.000000 & 0.000000 & 0.000000 \\
\hline
\end{tabular}

Table 5. Deflection $w$ for different $X, Y$ and $\beta=0.0, \theta=45^{\circ}$ and $a / b=1.5$ at initial time $0 . K$.

\begin{tabular}{ccccccccc}
\hline$X$ & \multicolumn{2}{c}{$Y=0.2$} & \multicolumn{2}{c}{$Y=0.4$} & \multicolumn{2}{c}{$Y=0.6$} & \multicolumn{2}{c}{$Y=0.8$} \\
\cline { 2 - 9 } & First Mode & Second Mode & First Mode & Second Mode & First Mode & Second Mode & First Mode & Second Mode \\
\hline 0.0 & 0.000000 & 0.000000 & 0.000000 & 0.000000 & 0.000000 & 0.000000 & 0.000000 & 0.000000 \\
0.2 & 0.001055 & 0.000390 & 0.001365 & 0.000372 & 0.000201 & 0.000146 & 0.001580 & 0.002571 \\
0.4 & 0.002296 & 0.000051 & 0.002953 & -0.000392 & 0.000444 & 0.000270 & 0.003679 & 0.007028 \\
0.6 & 0.002296 & 0.000051 & 0.002953 & -0.000392 & 0.000444 & 0.000270 & 0.003679 & 0.007028 \\
0.8 & 0.001055 & 0.000390 & 0.001365 & 0.000372 & 0.000201 & 0.000146 & 0.001580 & 0.002571 \\
1.0 & 0.000000 & 0.000000 & 0.000000 & 0.000000 & 0.000000 & 0.000000 & 0.000000 & 0.000000 \\
\hline
\end{tabular}

Table 6. Deflection $w$ for different $X, Y$ and $\beta=0.6, \theta=0^{\circ}$ and $a / b=1.5$ at initial time $0 . K$.

\begin{tabular}{cccccccccc}
\hline & \multicolumn{2}{c}{$Y=0.2$} & \multicolumn{2}{c}{$Y=0.4$} & \multicolumn{2}{c}{$Y=0.6$} & \multicolumn{2}{c}{$Y=0.8$} \\
\cline { 2 - 9 } & First Mode & Second Mode & First Mode & Second Mode & First Mode & Second Mode & First Mode & Second Mode \\
\hline 0.0 & 0.000000 & 0.000000 & 0.000000 & 0.000000 & 0.000000 & 0.000000 & 0.000000 & 0.000000 \\
0.2 & 0.001233 & 0.000392 & 0.001634 & 0.000380 & 0.000212 & 0.000150 & 0.001310 & 0.002565 \\
0.4 & 0.002910 & 0.000063 & 0.003870 & -0.000371 & 0.000493 & 0.000271 & 0.002760 & 0.007007 \\
0.6 & 0.002910 & 0.000063 & 0.003870 & -0.000371 & 0.000493 & 0.000271 & 0.002760 & 0.007007 \\
0.8 & 0.001233 & 0.000392 & 0.001634 & 0.000380 & 0.000212 & 0.000150 & 0.001310 & 0.002565 \\
1.0 & 0.000000 & 0.000000 & 0.000000 & 0.000000 & 0.000000 & 0.000000 & 0.000000 & 0.000000 \\
\hline
\end{tabular}

Table 7. Deflection $w$ for different $X, Y$ and $\beta=0.6, \theta=45^{\circ}$ and $a / b=1.5$ at initial time $0 . K$.

\begin{tabular}{ccccccccc}
\hline & \multicolumn{2}{c}{$Y=0.2$} & \multicolumn{2}{c}{$Y=0.4$} & \multicolumn{2}{c}{$Y=0.6$} & \multicolumn{2}{c}{$Y=0.8$} \\
\cline { 2 - 8 } & First Mode & Second Mode & First Mode & Second Mode & First Mode & Second Mode & First Mode & Second Mode \\
\hline 0.0 & 0.000000 & 0.000000 & 0.000000 & 0.000000 & 0.000000 & 0.000000 & 0.000000 & 0.000000 \\
0.2 & 0.001182 & 0.000391 & 0.001560 & 0.000376 & 0.000210 & 0.000150 & 0.001386 & 0.002570 \\
0.4 & 0.002731 & 0.000061 & 0.003610 & -0.00036 & 0.000481 & 0.000271 & 0.003022 & 0.007014 \\
0.6 & 0.002731 & 0.000061 & 0.003610 & -0.00036 & 0.000481 & 0.000271 & 0.003022 & 0.007014 \\
0.8 & 0.001182 & 0.000391 & 0.001560 & 0.000376 & 0.000210 & 0.000150 & 0.001386 & 0.002570 \\
1.0 & 0.000000 & 0.000000 & 0.000000 & 0.000000 & 0.000000 & 0.000000 & 0.000000 & 0.000000 \\
\hline
\end{tabular}


Table 8. Deflection $w$ for different $X, Y$ and $\beta=0.0, \theta=0^{\circ}$ and $a / b=1.5$ at time 5.K.

\begin{tabular}{ccccccccc}
\hline \multirow{2}{*}{$X$} & \multicolumn{2}{c}{$Y=0.2$} & \multicolumn{2}{c}{$Y=0.4$} & \multicolumn{2}{c}{$Y=0.6$} & \multicolumn{2}{c}{$Y=0.8$} \\
\cline { 2 - 8 } & First Mode & Second Mode & First Mode & Second Mode & First Mode & Second Mode & First Mode & Second Mode \\
\hline 0.0 & 0.000000 & 0.000000 & 0.000000 & 0.000000 & 0.000000 & 0.000000 & 0.000000 & 0.000000 \\
0.2 & 0.001035 & 0.000325 & 0.001344 & 0.000313 & 0.000193 & 0.000123 & 0.001462 & 0.002141 \\
0.4 & 0.002287 & 0.000044 & 0.002962 & -0.000322 & 0.000431 & 0.000225 & 0.003361 & 0.005850 \\
0.6 & 0.002287 & 0.000044 & 0.002962 & -0.000322 & 0.000431 & 0.000225 & 0.003361 & 0.005850 \\
0.8 & 0.001035 & 0.000325 & 0.001344 & 0.000313 & 0.000193 & 0.000123 & 0.001462 & 0.002141 \\
1.0 & 0.000000 & 0.000000 & 0.000000 & 0.000000 & 0.000000 & 0.000000 & 0.000000 & 0.000000 \\
\hline
\end{tabular}

Table 9. Deflection $w$ for different $X, Y$ and $\beta=0.0, \theta=45^{\circ}$ and $a / b=1.5$ at time 5.K.

\begin{tabular}{cccccccccc}
\hline & \multicolumn{2}{c}{$Y=0.2$} & \multicolumn{2}{c}{$Y=0.4$} & \multicolumn{2}{c}{$Y=0.6$} & \multicolumn{2}{c}{$Y=0.8$} \\
\cline { 2 - 9 } & First Mode & Second Mode & First Mode & Second Mode & First Mode & Second Mode & First Mode & Second Mode \\
\hline 0.0 & 0.000000 & 0.000000 & 0.000000 & 0.000000 & 0.000000 & 0.000000 & 0.000000 & 0.000000 \\
0.2 & 0.000980 & 0.000292 & 0.001267 & 0.000280 & 0.000185 & 0.000111 & 0.001465 & 0.001940 \\
0.4 & 0.002131 & 0.000040 & 0.002741 & -0.000294 & 0.000412 & 0.000202 & 0.003414 & 0.005295 \\
0.6 & 0.002131 & 0.000040 & 0.002741 & -0.000294 & 0.000412 & 0.000202 & 0.003414 & 0.005295 \\
0.8 & 0.000980 & 0.000292 & 0.001267 & 0.000280 & 0.000185 & 0.000111 & 0.001465 & 0.001940 \\
1.0 & 0.000000 & 0.000000 & 0.000000 & 0.000000 & 0.000000 & 0.000000 & 0.000000 & 0.000000 \\
\hline
\end{tabular}

Table 10. Deflection $w$ for different $X, Y$ and $\beta=0.6, \theta=0^{\circ}$ and $a / b=1.5$ at time 5.K.

\begin{tabular}{cccccccccc}
\hline & \multicolumn{2}{c}{$Y=0.2$} & \multicolumn{2}{c}{$Y=0.4$} & \multicolumn{2}{c}{$Y=0.6$} & \multicolumn{2}{c}{$Y=0.8$} \\
\cline { 2 - 9 } & First Mode & Second Mode & First Mode & Second Mode & First Mode & Second Mode & First Mode & Second Mode \\
\hline 0.0 & 0.000000 & 0.000000 & 0.000000 & 0.000000 & 0.000000 & 0.000000 & 0.000000 & 0.000000 \\
0.2 & 0.001162 & 0.000311 & 0.001541 & 0.000301 & 0.000201 & 0.000115 & 0.001231 & 0.002030 \\
0.4 & 0.002740 & 0.000051 & 0.003645 & -0.000294 & 0.000465 & 0.000213 & 0.002600 & 0.005541 \\
0.6 & 0.002740 & 0.000051 & 0.003645 & -0.000294 & 0.000465 & 0.000213 & 0.002600 & 0.005541 \\
0.8 & 0.001162 & 0.000311 & 0.001541 & 0.000301 & 0.000201 & 0.000115 & 0.001231 & 0.002030 \\
1.0 & 0.000000 & 0.000000 & 0.000000 & 0.000000 & 0.000000 & 0.000000 & 0.000000 & 0.000000 \\
\hline
\end{tabular}

Table 11. Deflection $w$ for different $X, Y$ and $\beta=0.6, \theta=45^{\circ}$ and $a / b=1.5$ at time 5.K.

\begin{tabular}{ccccccccc}
\hline & \multicolumn{2}{c}{$Y=0.2$} & \multicolumn{2}{c}{$Y=0.4$} & \multicolumn{2}{c}{$Y=0.6$} & \multicolumn{2}{c}{$Y=0.8$} \\
\cline { 2 - 8 } & First Mode & Second Mode & First Mode & Second Mode & First Mode & Second Mode & First Mode & Second Mode \\
\hline 0.0 & 0.000000 & 0.000000 & 0.000000 & 0.000000 & 0.000000 & 0.000000 & 0.000000 & 0.000000 \\
0.2 & 0.001080 & 0.000274 & 0.001420 & 0.000263 & 0.000191 & 0.000102 & 0.001261 & 0.001792 \\
0.4 & 0.002490 & 0.000042 & 0.003283 & -0.000263 & 0.000436 & 0.000190 & 0.002752 & 0.004896 \\
0.6 & 0.002490 & 0.000042 & 0.003283 & -0.000263 & 0.000436 & 0.000190 & 0.002752 & 0.004896 \\
0.8 & 0.001080 & 0.000274 & 0.001420 & 0.000263 & 0.000191 & 0.000102 & 0.001261 & 0.001792 \\
1.0 & 0.000000 & 0.000000 & 0.000000 & 0.000000 & 0.0000000 & 0.000000 & 0.000000 & 0.000000 \\
\hline
\end{tabular}


On comparison with [13], it is concluded that:

Time period $K$ is more for non-uniform thickness in case of parabolic variation as comparison to linear variation.

Deflection $w$ is less for non-uniform thickness in case of parabolic variation as comparison to linear variation.

In this way, authors concluded that parabolic variation is more useful than linear variation.

\section{References}

[1] M. S. Dhotarad and N. Ganesan, "Vibration Analysis of a Rectangular Plate Subjected to a Thermal Gradient," Journal of Sound and Vibration, Vol. 60, No. 4, 1978, pp. 481-497.

[2] M. Amabili and R. Garziera, "Transverse Vibrations of Circular, Annular Plates with Several Combinations of Boundary Conditions," Journal of Sound and Vibration, Vol. 228, No. 2, 1999, pp. 443-446.

[3] S. Ceribasi and G. Altay, "Free Vibration of Super Elliptical Plates with Constant and Variable Thickness by Ritz Method," Journal of Sound and Vibration, Vol. 319, No. 1-2, 2009, pp. 668-680.

[4] U. S. Gupta, A. H. Ansari and S. Sharma, "Vibration Analysis of Non-Homogenous Circular Plate of NonLinear Thickness Variation by Differential Quadrature Method," Journal of Sound and Vibration, Vol. 298, No. 4-5, 2006, pp. 892-906.

[5] R. K. Jain and S. R. Soni, "Free Vibrations of Rectangular Plates of Parabolically Varying Thicknesses," Indian Journal of Pure and Applied Mathematics, Vol. 4, No. 3, 1973, pp. 267-277.

[6] B. Singh and V. Saxena, "Transverse Vibration of Rec- tangular Plate with Bidirectional Thickness Variation," Journal of Sound and Vibration, Vol. 198, No. 1, 1996, pp. 51-65.

[7] J. S. Tomar, D. C. Gupta and V. Kumar, "Free Vibrations of Non-Homogeneous Circular Plate of Variable Thickness Resting on Elastic Foundation," Journal of Engineering Design, Vol. 1, No. 3, 1983, pp. 49-54.

[8] J. S. Yang, "The Vibration of a Circular Plate with Varying Thickness," Journal of Sound and Vibration, Vol. 165, No. 1, 1993, pp. 178-184.

[9] U. S. Gupta, A. H. Ansari and S. Sharma, "Vibration of Non-Homogeneous Circular Mindlin Plates with Variable Thickness," Journal of Sound and Vibration, Vol. 302, No. 1-2, 2007, pp. 1-17.

[10] D. V. Bambill, C. A. Rossit, P. A. A. Laura and R. E. Rossi, "Transverse Vibrations of an Orthotropic Rectangular Plate of Linearly Varying Thickness and with a Free Edge," Journal of Sound and Vibration, Vol. 235, No. 3, 2000, pp. 530-538.

[11] A. W. Leissa, "Recent Research in Plate Vibrations: Classical Theory," The Shock and Vibration Digest, Vol. 9, No. 10, 1977, pp. 13-24.

[12] Z. Sobotka, "Free Vibration of Visco-Elastic Orthotropic Rectangular Plates," Acta Technica (Czech Science Advanced Views), No. 6, 1978, pp. 678-705.

[13] A. K. Gupta, A. Kumar and Y. K. Gupta, "Vibration Study of Visco-Elastic Parallelogram Plate of Linearly Varying Thickness," International Journal of Engineering and Interdisciplinary Mathematics, accepted for publication.

[14] K. Nagaya, "Vibrations and Dynamic Response of Viscoelastic Plates on Non-Periodic Elastic Supports," Journal of Engineering for Industry, Vol. 99, 1977, pp. 404-409. 


\section{Appendix: List of Symbols}

$\begin{array}{lll}a & \text { length of the plate, } & \check{n} \\ b & \text { width of the plate, } & w(\xi, \eta, t) \\ \xi \text { and } \eta & \text { co-ordinates in the plane of the plate, } & W(\xi, \eta) \\ h & \text { thickness of the plate at the point }(\xi, \eta), & T(t) \\ E & \text { young's modulus, } & \beta \\ G & \text { shear modulus, } & K \\ v & \text { Poisson's ratio, } & h_{0} \\ \widetilde{D} & \text { visco-elastic operator, } & a / b \\ D & E h^{3} / 12\left(1-v^{2}\right), \text { flexural rigidity, } & \theta \\ \rho & \text { mass density per unit volume of the } & T_{\max } \\ t & \text { plate material, } & V \\ & \text { time, } & \lambda^{2}\end{array}$

Visco-elastic constants,

deflection of the plate i.e. amplitude,

deflection function,

time function,

taper constant,

time period,

$h$ at $\xi=0$,

aspect ratio,

skew angle,

Kinetic energy,

Strain energy,

$12 \rho\left(1-v^{2}\right) \mathrm{a}^{4} / E h_{0}{ }^{2}$, a frequency parameter 